\title{
Acceptability of a web-based dietary assessment tool for an Irish adult population: Foodbook24
}

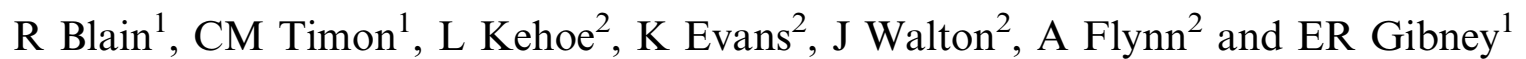 \\ ${ }^{1}$ Institute of Food and Health, University College Dublin, Belfield, Dublin 4, Ireland and ${ }^{2}$ School of Food and \\ Nutritional Sciences, University College Cork, Cork, Ireland.
}

Use of technology may offer possible solutions to known limitations associated with dietary assessment methodologies such as cost, participant or researcher burden, and coding of data. Foodbook $24^{(1)}$ (FB24) is a web-based 24 hour dietary recall tool which has been developed for use in the Irish adult population. The aim of this project was to gather user acceptability data on the tool using an online evaluation questionnaire.

FB24 is based on the USDA ${ }^{(2)}$ multiple pass recall method and has been developed in Ireland as a collaboration between University College Dublin and University College Cork. The tool has been used by 120 participants across two studies. The first study investigated the comparability of FB24 relative to an interview-led 24 hour dietary recall. The second study investigated the validity of FB24 using nutritional biomarkers against a gold standard dietary assessment method - a semi-weighed food diary. Upon completion of each study, participants were emailed a 16 item online evaluation questionnaire hosted on Google forms. The questionnaire consisted of both closed and open questions and were identical for each study except for one question pertaining to the preferred method of assessment; FB24 vs. semi-weighed food diary and FB24 vs. interview-led 24 hour dietary recall. The design of each questionnaire was based around those used in similar studies investigating user acceptability of technology based dietary assessment tools ${ }^{(3)}$.

In total 118 participants (58 male, 60 female) completed the optional evaluation questionnaire (78 from the comparison study and 40 from the validation study). Frequencies of responses were obtained using SPSS (IBM Version 20.0). The majority of respondents were positive in their evaluation questionnaire with a combined $69.5 \%$ 'satisfied' with using the tool. Only a minority of $7.6 \%$ found 'adding or removing food and drink items difficult'. Over $95 \%$ were happy with the visual presentation (text, buttons), while users found the time spent completing a recall to be OK $(63.6 \%)$ or short $(22.9 \%)$ with only a small number claiming it was too long $(6.8 \%)$. When asked if users felt using FB24 changed the food or drinks they ate a majority felt it did not change at all $(62.7 \%)$ while some felt it changed a little $(34.7 \%)$ and a small few felt it changed a lot $(2.5 \%)$. The majority of users reported no concerns about recording all food and drinks $(95.8 \%)$ using the tool. In terms of repeated use $82 \%$ reported being willing to use it for a week, with a smaller number stating they would be willing to use it for a month (55.1\%) or six months (24.6\%). Importantly, when asked which method users would prefer to use in future when reporting their dietary intake $67.8 \%$ opted for FB24 instead of the traditional face-to-face recall method. When responses were split by study involvement, FB24 was still the preferred method against the interview-led recall $(61.5 \%)$ and an even clearer favourite against the semi-weighed food diary $(80 \%)$. These findings on preference of method are in line with similar studies on acceptability of technology based dietary assessment methods ${ }^{(3,4)}$.

Overall FB24 was received well among users across both studies with a large proportion stating they would be willing to use the tool for longer than a week. The overall preference for FB24 over traditional methods is encouraging and may highlight individuals' willingness to move towards web-based means of dietary intake reporting.

The project is funded by the Irish Department of Agriculture, Food and the Marine under the Diet Ireland project $13 \mathrm{~F} 424$.

1. University College Dublin and University College Cork. Foodbook24 web based tool. 2016. Available from: www.foodbook24.com

2. Moshfegh AJ, Rhodes DG, Baer DJ, et al. (2008) Am J Clin Nutr, 88(2): 324-32.

3. Vereecken CA, Covents M, Haynie D, et al. (2009) J Am Diet Assoc,109(11): 1896-1902.

4. Monnerie B, Tavoularis LG, Guelinckx I et al. (2015) Eur J Nutr, 54: 27-34. 